\title{
Prevalência de obesidade em crianças e adolescentes com diabetes melito tipo 1
}

\author{
Prevalence of obesity in children and adolescents with type 1 diabetes mellitus
}

Raphael Del Roio Liberatore Junior ${ }^{1}$, Adriane de André Cardoso-Demartini², Allan Hiroshi de A. Ono ${ }^{3}$, Gabriel Costa de Andrade ${ }^{3}$,

\section{RESUMO}

Objetivo: Conhecer a prevalência de obesidade e sua distribuição por sexo e idade em pacientes com diabetes melito tipo 1 (DM1), em acompanhamento em Serviço de Endocrinologia Pediátrica.

Métodos: Estudo transversal descritivo de pacientes em acompanhamento no Serviço de Endocrinologia Pediátrica do Hospital de Base de São José do Rio Preto, avaliando as seguintes variáveis: sexo, idade, dose e número de aplicações diárias de insulina, hemoglobina glicada $\left(\mathrm{HbA}_{1 \mathrm{c}}\right)$, peso, estatura e índice de massa corpórea (IMC). A definição de sobrepeso e obesidade baseou-se nas curvas de IMC para a idade do Centers for Disease Control and Prevention (CDC, 2000).

Resultados: Foram incluídos 77 portadores de DM1 (44 meninas e 33 meninos) com idade entre três e 18 anos (média: 13,4). A determinação de $\mathrm{HbA}_{1 \mathrm{c}}$ variou de 4,1 a 10,6\% (média: 7,7\%) e o IMC, de 15,1 a 28,6 kg/m² (média: 23,2). Conforme critérios do CDC, $8 \%$ dos pacientes (três meninas e três meninos) apresentavam obesidade e $8 \%$ (duas meninas e quatro meninos), sobrepeso.

Conclusões: Nesta casuística de pacientes diabéticos, a prevalência de sobrepeso e obesidade não se mostrou diferente de populações não diabéticas.

Palavras-chave: obesidade; sobrepeso; índice de massa corpórea; diabetes mellitus tipo 1.

\section{ABSTRACT}

Objective: To describe the prevalence of obesity according to gender and age in patients with type 1 diabetes mellitus of a pediatric endocrinology follow-up clinic.

Methods: This cross-sectional descriptive study enrolled patients of the follow-up clinic of the Pediatric Endocrinology Unit of Hospital de Base of São José do Rio Preto, São Paulo, Brazil. The following data were collected: gender, age, insulin dosage, glycated hemoglobin $\left(\mathrm{HbA}_{1 \mathrm{c}}\right)$, weight, height and body mass index (BMI). The cutoff criteria for overweight and obesity were based on BMI-for-age growth charts of the Centers for Disease Control and Prevention (CDC, 2000).

Results: 77 patients with type 1 diabetes mellitus were included ( 44 females and 33 males), with three to 18 years old (mean: 13.4). The value of $\mathrm{HbA}_{1 \mathrm{c}}$ varied from 4.1 to $10.6 \%$ (mean: $7.7 \%$ ) and the BMI ranged from 15.1 to $28.6 \mathrm{~kg} / \mathrm{m}^{2}$ (average: 23.2 ). Based on BMI charts, $8 \%$ (three girls and three boys) were obese and $8 \%$ (two girls and four boys) had overweight.

Conclusion: Compared to the prevalence of obesity and overweight in non-diabetic pediatric population, the presence of these nutritional disturbances in the studied children was not significantly different.

Key-words: obesity; overweight; body mass index; diabetes mellitus, type 1 .
1Professor doutor, chefe do Serviço de Endocrinologia Pediátrica do Hospital de Base da Famerp, São José do Rio Preto, SP, Brasil

${ }^{2}$ Mestre em Pediatria, médica voluntária do Serviço de Endocrinologia Pediátrica do Hospital de Base da Faculdade de Medicina de São José do Rio Preto (Famerp), São José do Rio Preto, SP, Brasil

${ }^{3}$ Aluno de Iniciação Científica da Famerp, São José do Rio Preto, SP, Brasil
Endereço para correspondência:

Raphael Del Roio Liberatore Junior

Rua Ondina, 54 - Redentora

CEP 15015-205 - São José do Rio Preto/SP

E-mail: liberat@famerp.br

Fonte financiadora do projeto: Bolsa de Iniciação Científica da Famerp 


\section{Introdução}

O termo diabetes melito (DM) aplica-se a um conjunto de desordens crônicas caracterizadas pelo metabolismo anormal de proteínas, gorduras e carboidratos. Essa situação decorre da falta da insulina ou de sua incapacidade em exercer seus efeitos. Como resultado, o indivíduo portador de DM apresenta hiperglicemia crônica e possibilidade de desenvolvimento, em longo prazo, de complicações microvasculares e macrovasculares ${ }^{(1)}$.

A incidência de obesidade está aumentando rapidamente em nossa sociedade e associa-se a várias comorbidades endócrinas e metabólicas, incluindo DM tipo 2, hipertensão arterial sistêmica e dislipidemia, além de colelitíase, osteoartrite, apnéia do sono e distúrbios psiquiátricos ${ }^{(2,3)}$. A obesidade é considerada como um fator de risco independente para o aumento da mortalidade ${ }^{(4)}$. A etiologia da obesidade é multifatorial, resultando da inadequação entre a ingestão calórica e o gasto energético, associada a fatores genéticos, ambientais e comportamentais, como sedentarismo, dietas desequilibradas e aumento da ingestão alimentar ${ }^{(5-7)}$.

Em geral, pacientes com DM tipo 1 (DM1) possuem índice de massa corpórea (IMC) normal ou até abaixo da normalidade. Já foi observado ganho excessivo de peso em portadores de DM1 em tratamento intensivo com insulina. Também foram notados níveis mais elevados de colesterol total, LDL-colesterol e triglicerídeos, além de valores menores de HDL-colesterol, apesar de um adequado controle glicêmico ${ }^{(8,9)}$.

Diante desse quadro, no qual a obesidade vem se transformando em importante problema de Saúde Pública, e frente à possibilidade de concomitância de diabetes e obesidade, o presente estudo visou analisar a prevalência de sobrepeso e obesidade em crianças e adolescentes portadores de DM1 em acompanhamento em serviço de referência no interior do Estado de São Paulo.

\section{Métodos}

Estudo de corte transversal e descritivo que avaliou prontuários de crianças e adolescentes em acompanhamento no Serviço de Endocrinologia Pediátrica do Hospital de Base, na cidade de São José do Rio Preto, São Paulo. O projeto de pesquisa foi aprovado pelo Comitê de Ética em Pesquisa da Faculdade de Medicina de São José do Rio Preto. Após a aprovação, iniciou-se a revisão de 141 prontuários de crianças e adolescentes de até 18 anos de idade, em seguimento no ambulatório, com diagnóstico E14.9, segundo a Classificação Internacional de Doenças, que corresponde à DM.
As seguintes variáveis foram coletadas: sexo, idade, peso, estatura, IMC, número de aplicações e dose diária de insulina e hemoglobina glicosilada $\left(\mathrm{HbA}_{1 c}\right)$ na última consulta. Dos 141 prontuários avaliados, 64 foram excluídos por não apresentarem todos os dados ou por erro na classificação diagnóstica.

O peso (P) e a estatura (E) de cada paciente foram obtidos com balança Filizola ${ }^{\circledR}$ e com um estadiômetro fixo em parede, respectivamente. O IMC foi calculado pela fórmula: $\mathrm{P}(\mathrm{kg}) /[\mathrm{E}(\mathrm{m})]^{2}$. A partir da curva de IMC para a idade do Centers for Disease Control and Prevention (CDC) ${ }^{(10)}$, os pacientes foram classificados em eutróficos (percentis 3-84), portadores de sobrepeso (percentis 85-94) e obesos (percentil $\geq 95)^{(6)}$. A dosagem de $\mathrm{HbA}_{1 \mathrm{c}}$ foi realizada por cromatografia líquida de alto desempenho, cujo intervalo de referência é de 4,6 a 6,5\%.

Os resultados foram armazenados em planilha eletrônica e apresentados como intervalo mínimo-máximo e mediana. A análise de variância (ANOVA) para amostras repetidas foi utilizada para verificar se, variando os valores de hemoglobina glicosilada e de aplicações e dose diária de insulina, ocorria interferência na variável IMC. Para avaliação estatística, foi utilizado o programa SigmaStat for Windows, versão 2.03. Considerou-se significante $p<0,05$.

\section{Resultados}

Foram selecionados 77 pacientes com diagnóstico de DM1. A idade variou de três a 18 anos (mediana: 13,4 anos), sendo 44 do sexo feminino e 33 do masculino. A dose de insulina variou de 0,6 a 1,2 unidades $/ \mathrm{kg} / \mathrm{dia}$, sendo que 69 $(89,6 \%)$ pacientes faziam duas aplicações diárias e o restante $(10,4 \%)$, apenas uma. Os valores de $\mathrm{HbA}_{1 \mathrm{c}}$ variaram de 4,1 a $10,6 \%$, com mediana de $7,7 \%$.

Dentre os 77 pacientes, 65 eram eutróficos ( 39 meninas e 26 meninos), seis tinham sobrepeso (duas meninas e quatro meninos) e seis eram obesos (três meninas e três meninos).

Para a análise estatística, os valores de IMC foram divididos em três intervalos distintos, a partir das curvas do $\mathrm{CDC}^{(10)}$ : eutróficos, sobrepeso e obesos. Os resultados de hemoglobina glicosilada foram divididos em quatro intervalos: 4,1-6,1; 6,2-8,2; 8,3-10,3; e > 10,3. O número de aplicações foi estratificado em dois intervalos e a dose de insulina em quatro intervalos de $0,2 \mathrm{unid} / \mathrm{kg} / \mathrm{dia}$. Calculou-se, então, as medianas dos dados para cada um dos intervalos e foi feita a análise de variância. Esta não mostrou associação de nenhuma das variáveis com o IMC. 


\section{Discussão}

O aumento da prevalência global da obesidade infantil é uma das maiores preocupações da Organização Mundial da Saúde (OMS). No entanto, a obesidade é um dos problemas de Saúde Pública mais negligenciados ${ }^{(11)}$. Em 2004, em Genebra, estimou-se que 22 milhões de crianças com menos de cinco anos de idade encontravam-se obesas ou com sobrepeso. Destas, mais de 17 milhões viviam em países em desenvolvimento ${ }^{(12)}$.

Na população norte-americana, de 1976 a 2002, a prevalência de sobrepeso em adultos aumentou de 47 para $65 \%$ e a de obesidade dobrou de 15 para $31 \%^{(13)}$. A prevalência de sobrepeso aumentou de 7,2 para 13,9\% em crianças de dois a cinco anos de idade e de 11 para $19 \%$ naquelas com seis a 11 anos. No mesmo período, a prevalência de sobrepeso aumentou de 11 para $17 \%$ em adolescentes ${ }^{(14)}$. O objetivo da OMS para 2010 é reduzir a prevalência de obesidade em adultos para níveis inferiores a $15 \%{ }^{(13)}$.

No Brasil, já foram realizados estudos mostrando o aumento da obesidade infantil. Em 2003, Leão et al ${ }^{(15)}$ encontraram prevalência de obesidade de $30 \%$ em crianças de cinco a 10 anos de idade na rede privada de ensino e em $8 \%$ das crianças de escolas públicas. Em estudo realizado em uma cidade nordestina brasileira ${ }^{(16)}$, a prevalência de sobrepeso e obesidade em crianças de cinco e nove anos foi de 9,3 e 4,4\%, respectivamente, não havendo diferença significante entre os sexos. Por sua vez, Abrantes et al ${ }^{(17)}$ encontraram prevalência de obesidade em adolescentes de 6,6\% no Nordeste e 8,4\% no Sudeste e, em crianças, de 8,2 e 11,9\% nas regiões Nordeste e Sudeste, respectivamente.

A definição de obesidade em crianças e adolescentes tem sido discutida. Embora o peso seja considerado um indicador de gordura corporal, crianças com o mesmo peso mas com estaturas diversas podem apresentar diferentes graus de adiposidade; o IMC ajusta apenas parcialmente o peso para a estatura $^{(18)}$. No entanto, o uso do IMC por idade, baseado em padrão internacional ${ }^{(19)}$, mostrou-se adequado para o diagnóstico de sobrepeso e obesidade na faixa etária pediátrica, apresentando boa concordância com a adiposidade ${ }^{(18)}$.

Acreditava-se que a criança portadora de DM1 não apresentava sobrepeso e que essa característica estaria associada à diabetes tipo $2^{(20)}$. Entretanto, a resistência à insulina também pode existir em pacientes portadores de DM1. Reinehr $e t a l^{(21)}$ mostraram que a prevalência de sobrepeso em crianças com DM1 resistentes à insulina não é maior que a prevalência em crianças sensíveis à insulina, baseando-se na dose diária de insulina por $\mathrm{kg}$ de peso corporal (ID $/ \mathrm{kg}$ ). Concluíram ainda que a dose diária de insulina por metro quadrado de superfície corporal $\left(\mathrm{ID} / \mathrm{m}^{2}\right)$ e a dose diária de insulina por peso ideal (ID/iW) são os padrões que melhor relacionam a influência do sobrepeso na resistência à insulina em diabéticos tipo $1^{(21)}$. Libman et al ${ }^{(22)}$ investigaram a prevalência de sobrepeso em crianças e adolescentes que realizavam tratamento de diabetes com insulina em dois períodos, de 1979 a 1989 e de 1990 a 1998, constatando que esse número triplicou no segundo período, seguindo a tendência da população, em geral, não diabética.

O presente estudo consistiu na avaliação da prevalência de sobrepeso e obesidade em pacientes com DM1. Os valores de $\mathrm{HbA}_{1 \mathrm{c}}$ variaram entre 4,1 e 10,6\%, com média de 7,7\%, condizendo com a realidade de uma população diabética razoavelmente controlada. A prevalência de sobrepeso e obesidade neste grupo foi de, respectivamente, 8 e $8 \%$, o que difere de estudos anteriores, como o de Moraes et al ${ }^{(23)}$, em que o risco de sobrepeso e obesidade em crianças e adolescentes foi de 13,8 e $3 \%$, respectivamente. No entanto, como no presente estudo houve coleta de dados de prontuários, não foi possível avaliar certas variáveis, como ingestão alimentar e sedentarismo.

A baixa prevalência de distúrbios nutricionais nos pacientes pediátricos avaliados na presente pesquisa pode ser explicada não somente pela influência de particularidades regionais e econômicas nos padrões de alimentação, atividade física e controle metabólico, mas, sobretudo, devido à adequada monitoração dos pacientes aqui avaliados quanto a dieta, atividades físicas e controle da insulinoterapia. No entanto, estas afirmações são especulativas, pois a coleta de dados foi retrospectiva, o que não permitiu o acesso a informações como escolaridade dos pais, renda familiar e nível de atividade física. Todavia, os achados de obesidade e sobrepeso neste grupo de pacientes com DM1 foram semelhantes aos encontrados por Abrantes et al ${ }^{(17)}$, em 2002, em população não-diabética do Sudeste brasileiro.

Dentre as limitações do estudo, destaca-se o fato de seu desenho transversal indicar a avaliação nutricional das crianças e adolescentes com o tipo 1 em um determinado instante, sem, no entanto, analisar a evolução longitudinal do ganho de peso e do controle metabólico. Outra limitação é o uso exclusivo do IMC. Talvez, dados adicionais de medida da relação cintura-quadril e demais medidas antropométricas poderiam complementar e enriquecer os resultados obtidos.

Pode-se concluir que a prevalência de sobrepeso e obesidade em crianças com DM1 encontrada no presente estudo parece refletir a tendência mundial de aumento de peso na população pediátrica em geral, não havendo indicações 
de um aumento mais expressivo dessa tendência no grupo estudado. De qualquer maneira, para o acompanhamento nutricional de pacientes com DM1, é fundamental o controle do ganho de peso.

\section{Referências bibliográficas}

1. Associacion Latino Americana de Diabete. Definicion y diagnóstico de la diabete mellitus. Rev Assoc Latino Am Diabete 2000;1:121-3.

2. Dickey RA, Bray GA. Introduction to 1998 revision. AACE/ACE Obesity Statement 1995;298-330.

3. World Health Organization. Obesity: preventing and managing the global epidemic. Geneva: WHO; 1997.

4. Douketis JD, Feightner JW, Attia J, Feldman WF. Periodic health examination, 1999 update: 1. Detection, prevention and treatment of obesity. Canadian Task Force on Preventive Health Care. CMAJ 1999;160:513-25.

5. Sherwin RS. Diabetes melito. In: Goldeman L, Bennet JC, editores. Cecil: Tratado de medicina interna. $21^{\text {a }}$ ed. Rio de Janeiro: Guanabara Koogan; 2001. p. 1405-30.

6. Must A, Dallal GE, Dietz WH. Reference data for obesity: 85th and 95th percentiles of body mass index (wt/ht2): a correction. Am J Clin Nutr 1991;54:773.

7. Oliveira FLC, Escrivão MAMS, Sarni ROS. Obesidade - aspectos nutricionais. In: Monte O, Longui CA, Calliari LE, Kochi C, editores. Endocrinologia para o pediatra. $3^{\mathrm{a}}$ ed. São Paulo: Atheneu; 2006. p. 415-27.

8. Autoria não referida. The effect of intensive treatment of diabetes on the development and progression of long-term complications in insulin-dependent diabetes mellitus. The Diabetes Control and Complications Research Group. N Engl J Med 1993;329:977-86.

9. Purnell JQ, Hokanson JE, Marcovina SM, Steffes MW, Cleary PA, Brunzell JD. Effect of excessive weight gain with intensive therapy of type 1 diabetes on lipid levels and blood pressure: results from the DCCT. Diabetes Control and Complications Trial. JAMA 1998;280:140-6.

10. National Center for Health Statistics (NCHS), Centers for Disease Control and Prevention (CDC) [homepage on the Internet]. Clinical growth charts, 2000. Available from: http://www.cdc.gov/nchs/about/major/nhanes/growthcharts/ clinical_charts.htm

11. World Health Organization Nutrition [homepage on the Internet]. Controlling the global obesity epidemic [cited 2004 Nov 11]. Available from: www.who.int/ nutrition/topics/obesity/en/index.html

\section{Agradecimentos}

Este trabalho contou com bolsas de Iniciação Científica da Famerp.
12. World Health Organization [homepage on the Internet]. Fight childhood obesity to help prevent diabetes, say WHO \& IDF [cited 2003 Mar 3]. Available from: www.who.int/mediacentre/news/releases/2004/pr81/en/index.html

13. Prevalence of overweight and obesity among adults: United States, 1999-2002. United States Department of Health and Human Services. Hyattsville, MD: National Center for Health Statistics; 2002.

14. Prevalence of overweight among children and adolescents: United States, 2003-2004. United States Department of Health and Human Services. Hyattsville, MD: National Center for Health Statistics; 2004.

15. Leão LSCS, Araújo LMB, Moraes LTLP. Prevalência de obesidade em escolares de Salvador, Bahia. Arq Bras Endocrinol Metab 2003;47:151-7.

16. Oliveira AMA, Cerqueira EMM, Oliveira AC. Prevalência de sobrepeso e obesidade infantil na cidade de Feira de Santana-BA: detecção na família $x$ diagnóstico clínico. J Pediatr (Rio J) 2003;79:325-8.

17. Abrantes MM, Lamounier JA, Colosimo EA. Prevalência de sobrepeso e obesidade em crianças e adolescentes das regiões Sudeste e Nordeste. J Pediatr (Rio J) 2002;78:335-40.

18. Giugliano R, Melo AL. Diagnosis of overweight and obesity in schoolchildren: utilization of the body mass index international standard. J Pediatr (Rio J) 2004;80:129-34

19. Cole TJ, Bellizzi MC, Flegal KM, Dietz WH. Establishing a standard definition for child overweight and obesity worldwide: international survey. BMJ 2000;320:1240-3.

20. Autoria não referida. Type 2 diabetes in children and adolescents. American Diabetes Association. Diabetes Care 2000;23:381-9.

21. Reinehr T, Holl RW, Roth CL, Wiesel T, Stachow R, Wabitsch M et al. Insulin resistance in children and adolescents with type 1 diabetes mellitus: relation to obesity. Pediatr Diabetes 2005;6:5-12.

22. Libman IM, Pietropaolo M, Arslanain SA, LaPorte RE, Becker DJ. Changing prevalence of overweight children and adolescents at onset of insulin-treated diabetes. Diabetes Care 2003;26:2871-5.

23. Moraes CM, Portella RB, Pinheiro VS, Oliveira MMS, Fuks AG, Cunha EF et al. Prevalência de sobrepeso e obesidade em pacientes com diabetes tipo 1. Arq Bras Endocrinol Metab 2003;47:667-83. 